\title{
South African Marine Envenomations and Poisonings as Managed Telephonically by the Tygerberg Poisons Information Centre: A 20-Year Retrospective Review
}

\author{
Carine J. Marks, MSc ${ }^{1}$; Daniël J. Van Hoving, $\mathrm{MMed}^{2}$; Cherylynn A. Wium, MSc ${ }^{1}$; Dmitrij Sachno, $\mathrm{MSc}^{1}$; \\ Catharina E. Du Plessis, BSc ${ }^{1}$; Helmuth Reuter, $\mathrm{PhD}^{1}$; Gert J. Muller, $\mathrm{PhD}^{1}$ \\ ${ }^{1}$ Division of Clinical Pharmacology, Stellenbosch University, Stellenbosch, South Africa; ${ }^{2}$ Division of Emergency Medicine, Stellenbosch \\ University, Stellenbosch, South Africa
}

Introduction-South Africa has an abundance of marine life, and the potential for hazardous exposure to marine life is high. To our knowledge, this is the first epidemiological review regarding marine toxicity that has ever been conducted in sub-Saharan Africa. The aim of this review was to investigate marine toxicology data as managed telephonically by the Tygerberg Poisons Information Centre.

Methods-Marine toxicology cases were retrospectively analyzed for a 20-y period (January 1, 1995 to December 31, 2014). Data were extracted from archived consultation forms. Descriptive statistics are presented, and post hoc analyses compared age, sex, province, and caller's background with severity and type of toxicology.

Results-A total of 311 calls involved 392 cases. Most calls involved adults ( $\mathrm{n}=317,81 \%)$ and males $(n=214,55 \%)$ and presented with no or minor symptoms $(n=242,62 \%)$. Poisoning from ingestion $(n=239 ; 61 \%)$ was more commonly encountered than was marine envenomation $(n=153 ; 39 \%)$, with paralytic shellfish poisoning $(\mathrm{n}=118 ; 30 \%)$, scombroid poisoning $(\mathrm{n}=93 ; 24 \%)$, and envenomation from stingrays $(n=36 ; 9 \%)$ and bluebottles $(n=33 ; 8 \%)$ occurring often. Healthcare professionals were more likely to consult for severe cases (odds ratio 3.3; 95\% CI 1.9-5.9) and poisoning-related cases (odds ratio $1.8 ; 95 \%$ CI $1.1-2.9$ ).

Conclusion-The proportion of marine-related toxicology cases was low. Telephonic consultations by healthcare professionals relating to poisoning were generally of a serious nature. The data can be used to drive public health awareness campaigns.

Keywords: venomous fish, scombroid fish exposure, paralytic shellfish exposure, seafood poisoning

\section{Introduction}

The long South African coastline has an abundance of marine life, with an enormous diversity and complexity of venoms and poisons in marine animals. The potential for hazardous exposure to marine life in South Africa is thus high.

Dangerous aquatic animals can be divided into 4 groups: those that bite, those that sting (envenomation), those that can deliver an electric shock, and those that

Corresponding author: Carine J. Marks, Stellenbosch University, Division of Clinical Pharmacology, 35 Central Drive, Cape Town, Western Cape 8005, South Africa; e-mail: carinem@sun.ac.za.

Submitted for publication May 2018.

Accepted for publication December 2018. are poisonous if eaten. ${ }^{1}$ Exposure to the first 3 groups is increasing as the popularity of water-based sports grows. South Africa is a popular tourist destination, and together with the local population, increasing numbers of people are exposed to marine life.

Marine poisoning from eating seafood is increasing globally. One contributing factor is the global trade in exotic foods, which has given rise to the occurrence of instances of tropical food poisoning, such as ciguatera and tetrodotoxism, outside endemic areas. ${ }^{2}$ Seafood restaurants, especially those specializing in sushi, are becoming extremely popular in South Africa, and the potential risk of poisoning has also increased.

To our knowledge, this is the first epidemiological review regarding marine envenomation and poisoning 
that has ever been conducted in sub-Saharan Africa. The aim of this study was to investigate marine toxicology data as managed telephonically by the Tygerberg Poisons Information Centre (PIC).

\section{Methods}

Marine toxicology cases that were processed and documented by the Tygerberg PIC (in real time) were retrospectively analyzed for a 20-y period (January 1, 1995 to December 31, 2014). This study was approved by the Health Research Ethics Committee of Stellenbosch University (Ref: N 17/05/055).

South Africa is situated on the southernmost tip of Africa and has a coastline of approximately $2500 \mathrm{~km}$. It is divided into 9 provinces and has 3 not-for-profit PICs (Figure 1). One of them, the Tygerberg PIC, is situated at Tygerberg Hospital in Cape Town, Western Cape province. The other 2 PICs are located at the Red Cross Children's War Memorial Hospital (Cape Town, Western Cape) and at the University of the Free State (Bloemfontein, Free State). The Tygerberg PIC operated independently until the end of 2014 and provided a free, 24-h telephonic poisons information consultation service to South African healthcare workers and members of the public. A dedicated emergency number was advertised nationally, and poisoning enquiries were dealt with by medical doctors, pharmacists, and medical researchers with a Bachelor of Science degree.

The Tygerberg PIC manually recorded case information on standard consultation forms, and these data were and still are used for toxicovigilance and research purposes. ${ }^{3}$ Data from the archived consultation forms were entered by the authors on a Microsoft Excel spreadsheet

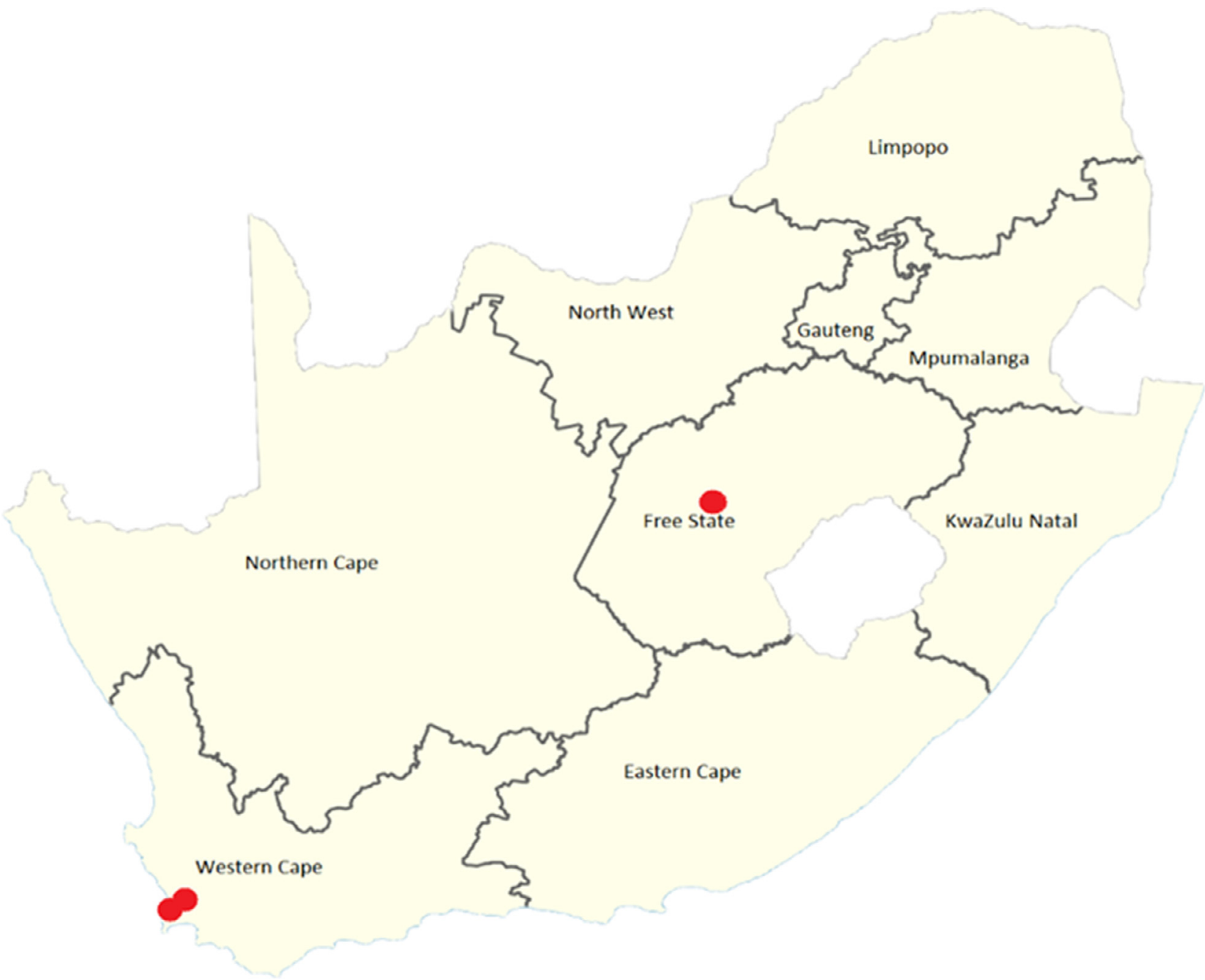

Figure 1. Map of South Africa, illustrating the 9 provinces and location of the 3 poisons information centers (red dots). 


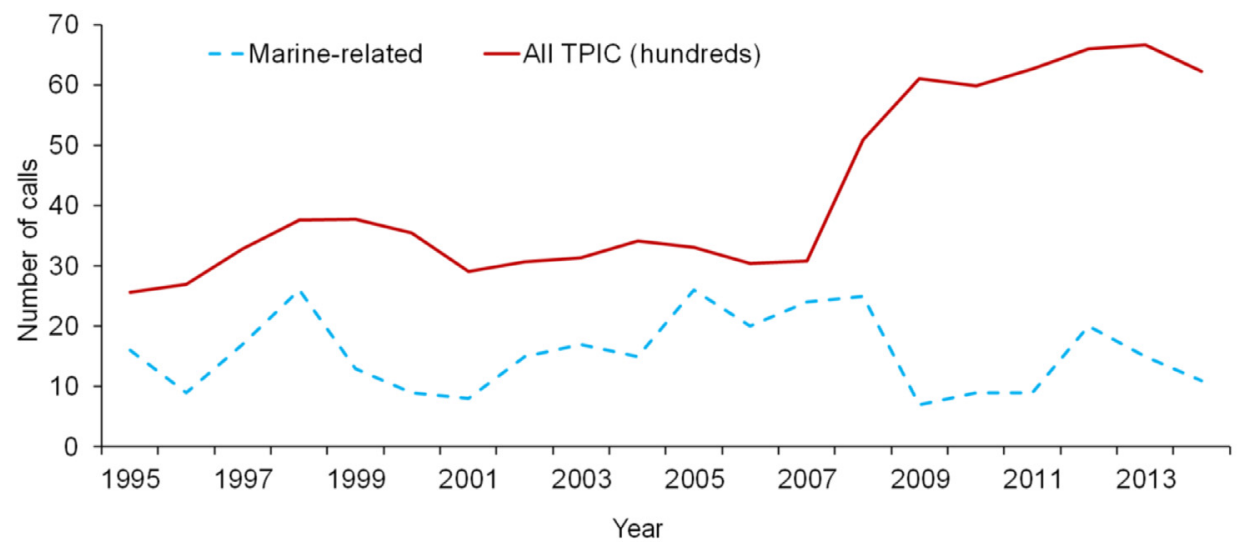

Figure 2. Trend of marine-related and total calls managed by the Tygerberg Poisons Information Centre over a 20-y period (total calls are in hundreds to fit the vertical scale).

and included date and time, geographical area, background of the caller, patient's age and sex, type of exposure, and the severity of the incident. In the event of an incomplete consultation form, the case was not excluded; instead, the specific missing variable was indicated as unknown. Marine animals responsible for the envenomation or poisoning were identified based on the history and information given by the caller. The poisoning severity score (PSS) of the European Association of Poisons Centers and Clinical Toxicologists was used to grade severity (severity 0 to $1=$ no to minor symptoms; severity 2 to $3=$ moderate to severe toxicity). ${ }^{4}$ The PSS was determined at the time of the call, taking into account the observed clinical symptoms and signs. However, the PSS was calculated retrospectively for consultations done before the publication of the PSS in 1998.

Descriptive statistics are presented for all variables. Age was collected as categorical data and was dichotomized for the analyses into adults (age $\geq 20 \mathrm{y}$ ) and youth (age $<20$ y). A severe case was defined as a PSS $\geq 2$, and type of exposure was divided into envenomation (marine bites or stings) and poisoning (ingestion). A post hoc decision was made to compare age, sex, province, and caller's background with severity and type of toxicology to determine any associations. The Pearson's $\chi^{2}$ test was used, and a 2 -sided $P$ value $<0.05$ was deemed significant. Effect size is presented as odds ratio (OR) with $95 \%$ CI. Analyses were done on the number of cases (not calls), using SPSS statistical software (IBM SPSS Statistics for Windows, Version 25.0. Armonk, NY).

\section{Results}

The Tygerberg PIC processed 84,558 telephonic consultations during the 20-year study period, of which 311
$(0.4 \%)$ were related to marine toxicology exposures (median per year 15, interquartile range 9-20) (Figure 2). Twenty-three calls involved more than 1 case, resulting in a total of 392 cases (including 7 cases in which animals were poisoned).

The majority of marine-related calls involved adults $(\mathrm{n}=317,81 \%$; animals excluded $\mathrm{n}=7$; unknown $\mathrm{n}=17)$ and males $(n=214,55 \%$; animals excluded $n=7$; unknown $\mathrm{n}=62$ ). Most calls were received from the Western Cape province ( $\mathrm{n}=304,78 \%)$, KwaZulu Natal $(n=27,7 \%)$, and the Eastern Cape province $(n=23,6 \%)$ (unknown $n=7,2 \%$; outside South Africa $n=2,1 \%$ ). Healthcare professionals $(n=260,66 \%)$ consulted the Tygerberg PIC more often than did the general public $(\mathrm{n}=107,23 \%)$ (unknown $\mathrm{n}=25,6 \%)$.

Poisoning from ingestion $(n=239,61 \%)$ was more commonly encountered than marine envenomation $(\mathrm{n}=153,39 \%)$, with substantially more cases recorded during summer (December to February) and autumn (March to May) (Figure 3).

The different categories of type of exposure are presented in Figure 4. Shellfish poisoning due to a red tide $(133 / 239,56 \%)$ accounted for most seafood poisonings. More cases of paralytic shellfish poisoning (PSP) (118/ $133,89 \%)$ occurred compared to diarrheic shellfish poisoning $(15 / 133,11 \%)$. In 1997, a massive red tide hit the west coast of the Western Cape province; 29 cases of PSP were recorded, and 2 adult females died. There was another major outbreak in 2005, when 23 cases of PSP were recorded. The Cape yellowtail (Seriola lalandii) was the primary fish species involved in scombroid poisoning $(61 / 93,66 \%)$; no serious complications were encountered. There were 3 cases of ingested tetrodotoxin-containing organs of the pufferfish (Tetraodontidae). Most of the recorded envenomation was caused by acanthotoxic fish $(80 / 153,52 \%)$, particularly stingrays 


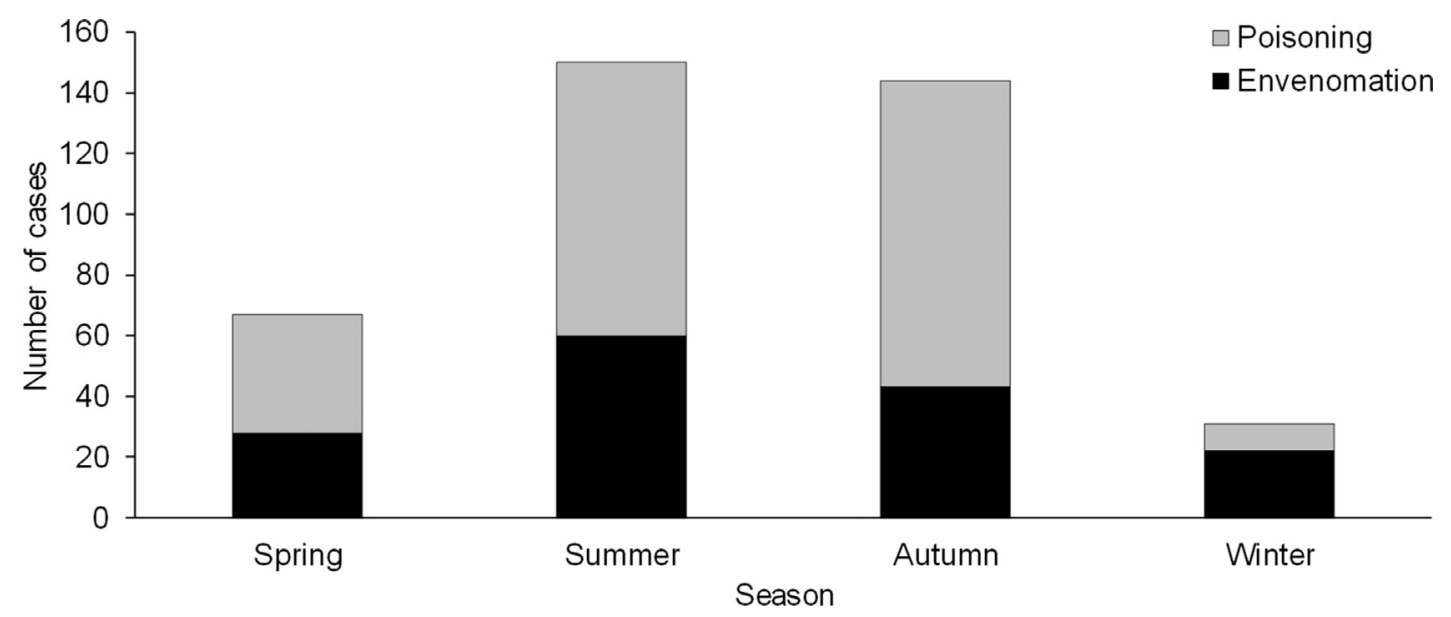

Figure 3. Seasonal distribution of calls related to marine envenomation and poisoning as reported to the Tygerberg Poisons Information Centre over 20 years.

$(36 / 80,45 \%)$ and sea barbells $(30 / 80,38 \%)$. The coelenterates were responsible for 48 cases $(31 \%)$ of marine envenomation, which were mainly caused by bluebottle stings $(33 / 48,69 \%)$.

Most patients $(n=242,62 \%)$ presented with no or minor symptoms (PSS 0 and 1) and reported local pain, minor local inflammatory reactions, and mild gastrointestinal symptoms. Moderate to severe toxicity (PSS 2 and 3) occurred in 129 patients (33\%). This group reported severe local pain, prominent local inflammatory reactions, prominent gastrointestinal effects, neurological toxic effects with difficulty in breathing, and respiratory failure. Four deaths $(1 \%)$ were recorded at the time of the call: An adult male died from an anaphylactic reaction after a bluebottle sting, 2 adult females died from respiratory failure after PSP, and $1 \mathrm{dog}$ died from tetrodotoxin poisoning after eating a puffer fish. Cases were not followed up, and no detail was available regarding the final outcome of the other cases.

Healthcare professionals were more likely than the general public to consult for severe cases (OR [95\% CI] $3.3[1.9-5.9]$ ) and poisoning-related cases (OR [95\% CI] 1.8 [1.1-2.9]) (Table 1). Significant associations were also found between poisoning-related cases and age, sex, and location (Table 1).

\section{Discussion}

This is the first study describing marine toxicology reported to a PIC in southern Africa. The incidence of marine-related telephonic consultations was relatively low compared to the overall call burden of the Tygerberg PIC (Figure 2). The overall number of calls to the Tygerberg PIC increased considerably during 2007, largely because of the closure of 2 other PICs in South Africa. A reason for the increase in enquiries may be that people call a PIC if they know one exists. ${ }^{3}$ Because of the closure of the PICs in KwaZulu-Natal and Gauteng, the Tygerberg PIC was contacted progressively more frequently. However, the number of marine cases reported during the same period did not increase exponentially. This skewed presentation of calls could be attributed to the inland geographical location of Gauteng, but it does not explain why there only was a slight increase in calls from KwaZulu-Natal, a coastal region.

The overall low incidence of reporting marine envenomation and poisoning-only $0.4 \%$ of the calls processed by the Tygerberg PIC during the study period-is similar to data from the United States and Australia., The American Association of Poison Control Centers reported an incidence of $0.1 \%$ (2079/2,159,000 during 2015), ${ }^{5}$ whereas the Queensland PIC reported marine exposures at an incidence of $0.4 \%(117 / 30,247)$ for 2016. ${ }^{6}$ Globally, many cases of mild marine poisoning or envenomation go unreported, and it is mostly the more severe cases that are recorded at PICs. Despite considerable underreporting, this number will likely rise in the future as a result of global warming, global trade, the internet, and the increase in popularity of eating exotic seafood. ${ }^{7,8}$

Adult males were significantly more likely to be involved in marine envenomation, and this is similar to previous findings. ${ }^{9,10}$ Most participants in water-related sports, such as diving, surfing, and fishing, are men, and they are thus more frequently exposed to potentially dangerous marine hazards. Additionally, men are also more inclined to engage in high-risk behavior, further increasing their likelihood of injury. ${ }^{11}$

The coastal provinces (Western Cape province, KwaZulu-Natal, Eastern Cape province), with the exception 


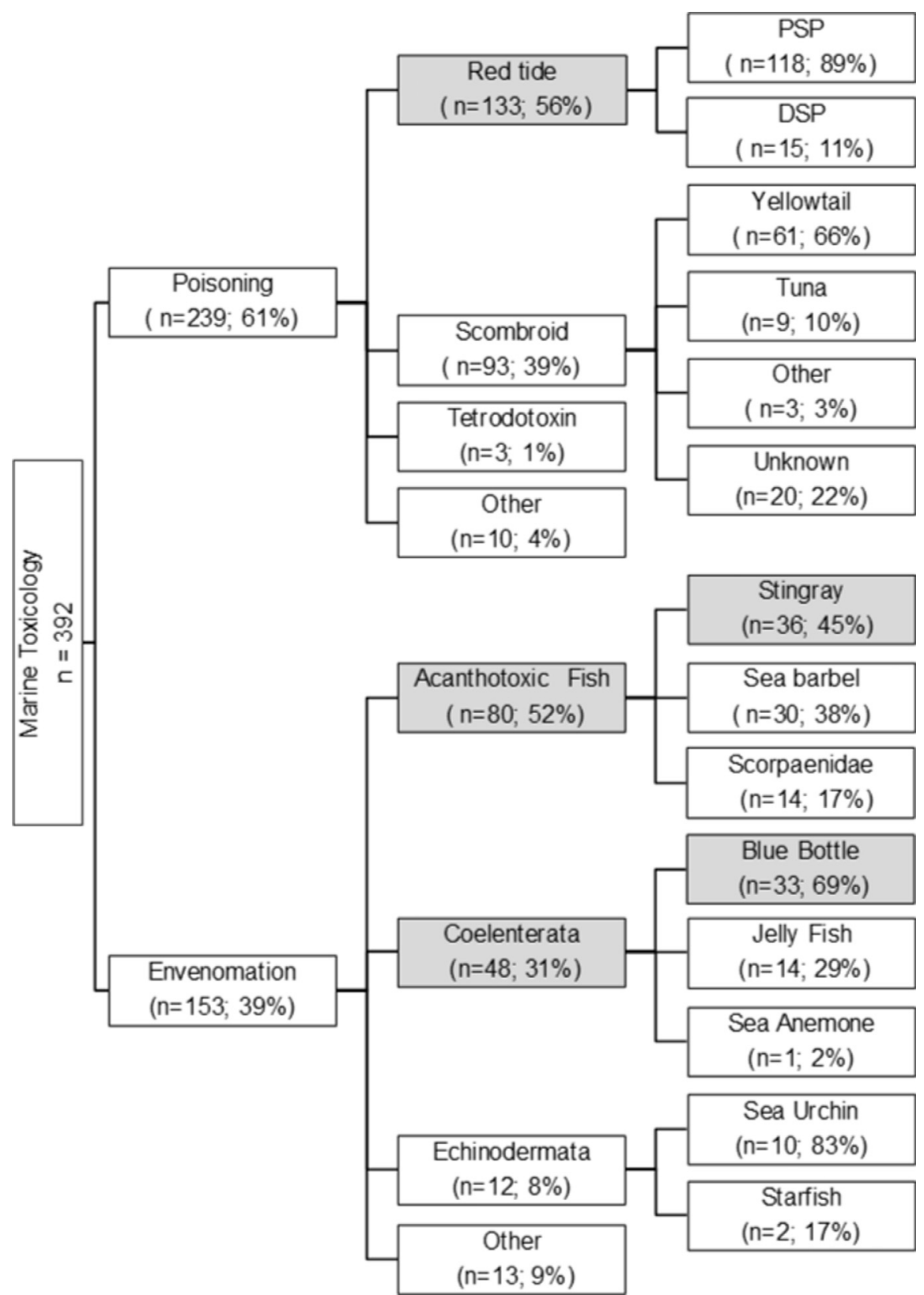

Figure 4. Different categories of marine toxicology exposure as reported to the Tygerberg Poisons Information Centre over $20 \mathrm{y}$ (more common reported exposures are represented by gray).

of the Northern Cape, had the most enquiries. This finding could well relate to the seasonal variation; people tend to visit coastal regions during the warmer months to participate in water sports or to relax on the beach. The low incidence witnessed in the Northern Cape province might be because it is the province with the lowest population density. Furthermore, the cold Benguela Sea current runs along the coastal stretch, making it less popular for water sports and thus a less-visited tourist area.
Compared to marine envenomation, marine poisoning cases reported to the Tygerberg PIC were more likely to be serious and occurred more often in adult females. Healthcare professionals consulted the Tygerberg PIC more frequently than did the general public, and these telephonic consultations were related to more serious cases. This intuitively makes sense: Members of the general public will probably only attend a healthcare service when they experience severe symptoms; likewise, the healthcare professional will seek advice if the clinical 
Table 1. Associations with severity and type of exposure in marine-related telephonic consultations at the Tygerberg Poisons Information Centre over a 20-y period

\begin{tabular}{lccccc}
\hline Variable & \multicolumn{2}{c}{ Severity $(P S S \geq 2)$} & & \multicolumn{2}{c}{ Type (poisoning) } \\
\cline { 2 - 3 } & $P$ & Odds ratio $(95 \%$ CI) & & $P$ & Odds ratio (95\% CI) \\
\hline Age $(\geq 20$ y) & 0.24 & $1.5(0.8-3.0)$ & & $<0.001$ & $5.4(2.8-10.6)$ \\
Sex (male) & 0.73 & $1.1(0.7-1.7)$ & & $<0.001$ & $0.3(0.2-0.5)$ \\
Location (Western Cape) & 0.7 & $1.1(0.7-1.9)$ & & $<0.001$ & $6.1(3.5-10.5)$ \\
Caller background (healthcare professional) & $<0.001$ & $3.3(1.9-5.9)$ & & 0.01 & $1.8(1.1-2.9)$ \\
Severity (PSS $\geq 2)$ & NA & NA & & $<0.001$ & $3.1(1.9-5.0)$ \\
\hline
\end{tabular}

PSS, poison severity score; NA, not applicable.

presentation is deemed serious. This hypothesis could unfortunately not be tested because of the retrospective nature of the study and the limited clinical information available.

Consultations for PSP surged in 1997 (29 cases) and in 2005 ( 23 cases), both associated with a red tide. Patients started to experience a tingling sensation and spreading numbness in the lips, face, and neck soon after ingesting the contaminated shellfish ( $30 \mathrm{~min}$ to $2 \mathrm{~h}$ ). Some patients also reported a prickly sensation in the fingertips and toes. Visual disturbances, vertigo, dizziness, ataxia, headache, a floating or gliding sensation, nystagmus, dysphagia, and dysarthria were further reported. The Tygerberg PIC advised healthcare professionals to treat all patients symptomatically and supportively. The symptoms usually cleared within 36 to $48 \mathrm{~h}$, but in 2005 , because of the consumption of heavily contaminated shellfish, 2 adult females developed progressive muscular paralysis and difficulty in breathing and died. Public health warnings during red tides should be increased and need to clearly stipulate the associated dangers of eating shellfish.

Scombroid poisoning, a mild to moderate form of food poisoning, occurred often and is linked to the consumption of "spoiled" fish as a result of improper storage conditions. Similar to previous data, the Cape yellowtail (Seriola lalandii) was the primary fish species involved. ${ }^{12}$ One possible reason for the relatively high occurrence of scombroid poisoning might be linked to the uniquely South African tradition of serving pickled fish on Good Friday. This is in line with our finding that most marine poisonings occurred in autumn (March to May). The increase in demand for fish during this period could well relate to a break in the cold chain from vendors and improper refrigeration due to poor socioeconomic situations on the user side. Furthermore, toxins produced during scombroid poisoning are heat stable and will not be destroyed by cooking or steaming. Further data are needed to investigate and confirm this possible association.
The study has various limitations, and care should be taken when generalizing the findings. First, the data reported are telephonic consultations by a poison center and may not reflect the true incidence and spectrum of marine-related toxicology cases in South Africa. One can assume that the total number of cases reported to the Tygerberg PIC is underrepresented, considering many healthcare professionals and even the general public might be familiar with marine envenomation and poisonings and would therefore not routinely call a poison center. The retrospective nature of this study also limits the significance of the clinical information. Some data were incomplete and might have influenced the results in either direction. However, this possible effect was diminished by indicating the specific missing variables as unknown and not excluding the entire case. Most calls were received from the Western Cape province and could have biased the study. Lastly, as a result of limited resources, cases were not followed up during the study period, and the study would have been substantially strengthened if data regarding patient-centered outcomes were available.

\section{Conclusion}

This is the first description of the epidemiology of marine-related toxicology cases from a South African PIC. The relative burden of these cases (in relation to the total number of calls managed) was low and mostly related to adults. Adult females were more likely to be involved in poisoning from seafood ingestion; in contrast, adult males were significantly more likely to be involved in marine envenomation. Healthcare professionals consulted significantly more often in serious cases compared with the general public, and envenomation was in general less serious than poisoning. The ability to identify and manage marine-related toxicology cases is important for providers of acute care, and poison centers should be contacted for telephonic advice on local marine envenomation and poisonings. Future studies 
should focus on robust data collection, including followup calls and patient-centered outcome data.

Author Contributions: Study concept and design (CJM, HR); acquisition of data (DS, CW, CD); analysis of data (CJM, DJVH); drafting of the manuscript (CJM, DJVH); critical revision of the manuscript (GM, DJVH); approval of final manuscript (CJM, DJVH, CW, DS, CD, HR, GM).

Financial/Material Support: None.

Disclosures: None.

\section{References}

1. Auerbach PS. Marine envenomations. $N$ Engl J Med. 1991;325(7):486-93.

2. Schmitt C, De Haro L. Clinical marine toxicology: a European perspective for clinical toxicologists and poison centers. Toxins (Basel). 2013;5(8):1343-52.

3. Veale DJH, Wium CA, Müller GJ. Toxicovigilance I: a survey of acute poisonings in South Africa based on Tygerberg Poison Information Centre data. S Afr Med J. 2013;103(5):293-7.

4. Persson HE, Sjöberg GK, Haines JA, Pronczuk de Garbino J. Poisoning severity score. Grading of acute poisoning. J Toxicol Clin Toxicol. 1998;36(3):205-13.

5. Mowry JB, Spyker DA, Brooks DE, Zimmerman A, Schauben JL. 2015 Annual report of the American Association of Poison Control Centers' National Poison Data System
(NPDS): 33rd annual report. Clin Toxicol (Phila). 2016;54 (10):924-1109.

6. Queensland Government. Children's Health Queensland Hospital and Health Service. Queensland Poisons Information Centre. Available at: www.childrens.health.qld.gov. $\mathrm{au} / \mathrm{chq} /$ our-services/queensland-poisons-information-cen tre/. Accessed August 15, 2018.

7. Schmitt C, De Haro L. Clinical marine toxicology: a European perspective for clinical toxicologists and poison centers. Toxins (Basel). 2013;5(8):1343-52.

8. Toovey S. Travelling to Africa: health risks reviewed. Travel Med Infect Dis. 2006;4(3-4):147-58.

9. Chan H, Chan Y, Tse M, Lau F, Kong H, Cheung CY. Venomous fish sting cases reported to Hong Kong Poison Information Centre: a three-year retrospective study on epidemiology and management. Hong Kong J Emerg Med. 2010;17(1):40-4.

10. Diaz JH. Marine Scorpaenidae envenomation in travelers: epidemiology, management, and prevention. $J$ Travel Med. 2015;22(4):251-8.

11. Udry JR. Why are males injured more than females. Inj Prev. 1998;4(2):94-5.

12. Muller GJ, Lamprecht JH, Barnes JM, De Villiers RV, Honeth BR, Hoffman BA. Scombroid poisoning: case series of 10 incidents involving 22 patients. S Afr Med J. 1992;81(8):427-30. 